•综述・

\title{
主流分子钟定年方法的原理、误差来源和使用建议
}

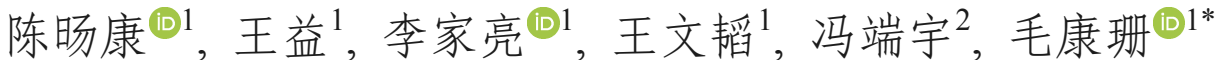

1. 四川大学生命科学学院生物资源与生态环境教育部重点实验室, 成都 $610065 ; 2$. 四川大学数学学院, 成都 610065

摘要: 近年来, 分子钟定年方法(molecular dating methods)得以广泛运用, 为宏观进化研究尤其是生物多样性及其格局形成历 史的相关研究提供了不可或缺且十分详尽的进化时间框架。贝叶斯方法(Bayesian methods)和马尔可夫链蒙特卡罗方法 (Markov chain Monte Carlo) 可容纳多维度、多类型的数据和参数设置, 因此以BEAST、PAML-MCMCTree等软件为代表的贝 叶斯节点标记法(Bayesian node-dating methods)逐渐成为分子钟定年方法中最为广泛使用的类型。贝叶斯框架的优势之一在于 其可以利用复杂模型考虑各种不确定性因素，但是该类方法中各类模型和参数的设置都可能引入误差，从而影响进化分化时 间估算的可靠性。本文介绍了贝叶斯分子钟定年方法的原理和主要类型，并以贝叶斯节点标记法为例，重点讨论了分子钟模 型、化石标记的选择与放置、采样频率及化石标记点年龄先验分布等因素对节点定年的影响; 提供了贝叶斯时间树构建软件 的使用建议、节点年龄的讨论原则和不同模型下时间树的比较方法，针对常见的引起节点年龄潜在高估和低估风险的情况作 了分析并给出了合理化建议。我们认为，合理整合多种贝叶斯方法和模型得出的结果并从中择优，能够提高定年结果的可靠 性; 研究人员应对时间树构建结果与其参数设置的关系开展讨论, 从而为其他学者提供参考; 化石记录的更新与分子钟定 年方法的改进应同步不断跟进。

关键词：分子钟；分子定年；贝叶斯节点标记法；系统发育；进化时间框架；分化时间

陈旸康，王益，李家亮，王文蹈，冯端宇，毛康珊 (2021) 主流分子钟定年方法的原理、误差来源和使用建议. 生物多样性，29，629-646. doi: 10.17520 /biods. 2020273

Chen YK, Wang Y, Li JL, Wang WT, Feng DY, Mao KS (2021) Principles, error sources and application suggestions of prevailing molecular dating methods. Biodiversity Science, 29, 629-646. doi: 10.17520/biods.2020273.

\section{Principles, error sources and application suggestions of prevailing molecular dating methods}

Yangkang Chen ${ }^{\mathbb{D}_{1}}$, Yi Wang ${ }^{1}$, Jialiang Li ${ }^{\left(\mathbb{D}_{1}\right.}$, Wentao Wang ${ }^{1}$, Duanyu Feng ${ }^{2}$, Kangshan Mao ${ }^{\text {(* }}$

1 Key Laboratory of Bio-resources and Eco-environment of Ministry of Education, College of Life Sciences, Sichuan University, Chengdu 610065

2 College of Mathematics, Sichuan University, Chengdu 610065

\begin{abstract}
Background \& Aims: Molecular dating methods have been applied widely in recent years and provide an indispensable and detailed evolutionary timescale for macroevolutionary researches, particularly for studies on the evolutionary history of biodiversity patterns. Bayesian methods and Markov chain Monte Carlo methods can accommodate multi-dimensional and various type of data and parameter settings, which have helped the node-dating methods implemented in softwares such as BEAST, PAML-MCMCTree to become the most widely used molecular dating methods. One of the advantages of Bayesian frameworks is that they can employ complex models to consider a variety of uncertainty factors to make more accurate estimations of evolutionary divergence times.

Progress: We review the principles and main types of Bayesian molecular dating methods and use Bayesian node-dating methods as an example to discuss potential errors in molecular clock models, selection and placement of fossil calibrating points, frequency of sampling, and setting a prior distribution for node calibrations based on fossils. We further describe advantages associated with different Bayesian time tree reconstruction software packages, the discussing principle of node age, and the comparison method of time tree under different models. We also provide suggestions for overcoming the challenges of overestimation and underestimation bias of node ages. Integration of the
\end{abstract}

收稿日期: 2020-07-08; 接受日期: 2020-08-18

基金项目：国家自然科学基金(31622015)和中央高校基础科研经费(SCU2019D013; SCU2018D006)

* 通讯作者 Author for correspondence. E-mail: maokangshan@scu.edu.cn 
output of various Bayesian methods and models and selection of the best among them often improve the reliability of molecular dating results.

Prospect: Researchers should explicitly assess the relationship between model output of time tree construction and model parameter settings, which increases transparency and provides documentation and reference for future researches. We recommend that future research simultaneously focus on updating fossil records and improving molecular dating methods.

Key words: molecular clock; molecular dating; Bayesian node-dating; phylogeny; evolutionary timescale; divergence age

分子钟定年方法(molecular dating approach)是 近年来宏观生物学尤其是生物多样性及其格局形 成历史相关研究最为重要的手段之一。该方法的基 础假设一一分子钟假设最早可以追溯至 20 世纪中 叶。1962年, Pauling和Zuckerkandl提出分子钟假设 (molecular clock hypothesis; Pauling \& Zuckerkandl, 1962), 随后Margoliash等(1963)对其进行了正式的 描述: “从两个物种最初分化开始, 它们的细胞色素 C残基(residue)序列之间的差异, 是由二者分化后 经历时间的长短决定的”。分子钟模型的核心原理 是: 在一定时间范围内, 特定生物大分子的序列在 不同类群间的差异与其分化后经历的时间呈近似 正线性关系。分子钟为进化生物学者提供了将微观 证据和宏观进化历史结合起来的、全新的重要方法 论。此前, 地球生命的进化历史重建主要结合化石 记录和地质历史进行, 但是由于化石埋藏形成的偶 然性等因素, 化石记录在不同类群中的稀缺程度大 不相同。事实上, 多数生物类群的化石记录, 尤其 是大化石记录(macrofossil) 十分稀缺 (Greenwood, 1991), 导致基于化石记录的进化历史研究较难开 展。以分子钟定年方法为代表的整合进化历史重建 方法, 可以同时考虑分子证据、形态证据、时间信 息等, 通过与分子系统发育与进化等相关的算法和 模型, 整合现生生物和古生物的进化历史数据 (Donoghue \& Moore, 2003), 形成一棵同时包含现 生生物和古生物类群的生命之树(tree of life), 从而 打破现生生物研究和古生物研究之间的藩篱, 更加 全面地揭示地球生物多样性形成的时间框架。

自1965年分子钟定年方法提出伊始, 许多学者 对这一方法提出质疑, 他们认为大分子序列的变化 是随机且无规律的(Morgan, 1998)。近二十多年来, 随着DNA测序技术的进步和各类模型算法的不断 完善, 分子钟定年方法不断进步, 在各个领域的应 用中认可度不断提高(Kumar, 2005; dos Reis et al, 2016)。如今分子钟已经被广泛用于生物学研究的各
个领域, 从体细胞突变、癌症细胞进化等微观研究 到生物多样性形成历史等宏观研究, 其提供的进化 时间框架不可或缺(Kumar \& Hedges, 2016; Graham \& Sottoriva, 2017)。因此，如何提高分子钟的可靠性 一直是进化生物学和生物地理学等研究领域的重 要议题。

利用分子钟模型进行系统发育树构建的方法 众多, 如贝叶斯法 (Bayesian)、最大似然法 (maximum likelihood, ML)、最大简约法(maximum parsimony, MP)、邻接法(neighbor-joining, $\mathrm{NJ}$ )等, 而 在时间树构建方面以贝叶斯法和最大似然法更为 可靠、主流、且衍生方法多样(Hall, 2005; Brooks et al, 2007; Yang \& Rannala, 2012)。由于贝叶斯方法在 20世纪末才被引入分子系统学领域, 国内介绍贝叶 斯框架下分子钟定年方法基本理论框架并提供使 用建议的文献仍存在一些空白。21世纪初, 有部分 国内学者较早地报道了贝叶斯方法在时间树构建 与物种分化时间估计中的应用(张原和陈之瑞, 2003), 后逐渐有学者对贝叶斯框架下分子钟假说 及其应用展开报道与论述(鲁丽敏等, 2014; 李可群, 2015; 刘晓枫和张爱兵, 2016; 朱天琪, 2019), 并提 供了各类贝叶斯软件的使用流程(鲁丽敏等, 2014; Zhang, 2019), 但对于复杂模型的参数及先验设置 的选择讨论较少, 且仍然缺少对于目前最常使用的 贝叶斯节点标记法(Bayesian node-dating method)程 序的详细介绍。

本文以贝叶斯节点标记法为例, 详细阐述了分 子钟定年方法的原理、误差来源, 以及如何规避这 些误差, 重点分析了多种情况下节点标记对分化时 间估计的影响, 并提供了合理的模型和软件使用建 议。

\section{1 贝叶斯节点标记法的基本原理和主要类型}

贝叶斯算法自 20 世纪末被引入分子系统发育 与进化领域以来, 随着计算效率的提升和马尔可夫 
链蒙特卡罗方法的引入(Markov chain Monte Carlo, MCMC; Yang \& Rannala, 1997; Chen \& Shao, 1999), 它在该领域的应用逐年递增, 目前已经成为分子系 统发育与进化领域中可信度和准确度最高的方法 之一(Yang \& Rannala, 2012; Brower, 2018)。贝叶斯 算法整合各项不确定性因素, 随着数据量的增加, 参数收玫到真值。对于每一个参数的概率分布, 观 测者都具有一定的经验认知, 即先验分布 (prior distribution), 体现了观测者经验的总结和主观预测 的结果。通过整合先验分布、给定数据集(data set) 以及其他参数, 可以得到各参数的后验分布 (posterior distribution)及均值, 体现了主观经验与客 观数据的结合。在数据量不足的情况下, 后验由先 验主导; 而当数据量逐渐累积, 先验产生的影响逐 渐减小, 后验将由客观数据主导 (Wasserman, 2004)。然而, 由于生物学数据的局限性, 即便使用 全基因组数据也往往难以使后验分布收敛到单一 真值, 因此先验分布的设置变得至关重要, 尤其是 在数据量较小的分析中(Yang \& Rannala, 2006)。

在分子钟模型下使用贝叶斯法进行计算时, 需 要设置序列数据 (sequence data) 、进化模型 (evolutionary model)和模型先验(prior; Bromham et al, 2018)。进化模型由核酸(蛋白质) 替换模型 (substitution model)和树模型(tree model)组成, 两类 模型又分别需要进行众多先验值及其分布的设定。 替换模型涉及的参数有碱基间替换速率(rate of transition between bases)、碱基频率 (base frequencies)、不同位点间替换速率(rate across sites) 的分布模型参数以及不变位点 (invariant sites)的比 例。树模型由树的拓扑 (topology) 和支长 (branch lengths) 两部分组成, 支长又由分支时长 (branch durations)和分支进化速率(branch rates)共同决定。 其中, 不同的参数可以施加不同的先验分布, 而对 于其中的一些先验分布的参数还可以进一步设置 超先验(hyperprior; Friston et al, 2013), 所有这些设 置构成了一个进化模型, 如图1所示。最终, 通过将 数据集应用于进化模型, 进行 MCMC的抽样和统计 分析, 得出时间树和各类参数的后验分布及可信区 间(credible intervals)。

在使用分子数据进行系统发育树的构建时, 尽 管分子序列数据的增加能够提高系统发育树的准 确度和精确度(dos Reis et al, 2012; Warnock et al,
2017), 但由于核酸(蛋白质)替换速率在不同进化分 支(evolutionary lineages)间的差异、不同地质时期古 环境因素导致进化速率的不同(Harwood et al, 2007; Laws, 2010)、世代时间各异(Thomas et al, 2010)等因 素, 只能得到系统发育树的拓扑以及相对物种分化 时间，而无法得到谱系间的绝对物种分化时间，即 只知道树的拓扑和相对支长 (遗传距离) 而不知绝对 支长。分支时长由树先验(tree prior)和校准信息 (calibration, 包括节点标记(node calibration)和端点 标记(tip calibration))共同决定, 在没有化石证据等 提供校准信息时, 仅依靠树先验得到相对年龄参考 价值较低。因此, 在贝叶斯框架下得到相对分化时 间后, 要进一步得到绝对分化时间, 最好的方法是 进行化石标定(fossil calibration; Barba-Montoya et al, 2017)。在获得某个类群的化石及其年龄后, 就可以 在相对分化时间框架的基础上整合化石年龄, 得出 进化时间树, 从而得出该类群进化树上各个节点的 绝对分化时间及其可信区间。

在贝叶斯框架下常用的化石标记方法包括节 点标记法 (node-dating)、全证据法 (total-evidence method; Ronquist et al, 2012)以及化石生灭进程法 (fossilized birth-death process; Heath et al, 2014)等。 节点标记法使用化石年龄数据标定系统发育树上 的相应节点; 全证据法和化石生灭进程法属于端点 标记法(tip-dating), 将化石视作系统发育树上的进 化分支进行树的构建, 充分利用了化石类群的形态 特征, 而不仅限于整合其年龄数据。三类方法各有 利弊(Grimm et al, 2015): 节点标记法虽然考虑了树 的结构和化石标记的不确定性(uncertainty), 但对化 石年龄先验 (作为时间先验) 的设置往往武断 (Sauquet, 2013), 只考虑使用年龄最老的化石, 且得 到的系统发育树中只包含现存类群; 全证据法基于 系统发育关系进行化石定位, 同时考虑了化石类群 和现存类群的定年, 但需要依赖化石及现存类群的 形态矩阵 (morphological matrix), 这一条件有时难 以满足, 且往往需要花费大量时间对现存物种的形 态学数据进行编码(Guillerme \& Cooper, 2016); 化 石生灭进程法能够运用所有的化石类群, 并且不需 要形态学矩阵, 但没有考虑到系统发育树的结构和 化石年龄的不确定性。根据其不同的特性, 以上三 种方法分别适用于化石记录贫乏的类群、形态性状 丰富的类群, 以及化石记录丰富的类群(Grimm et 


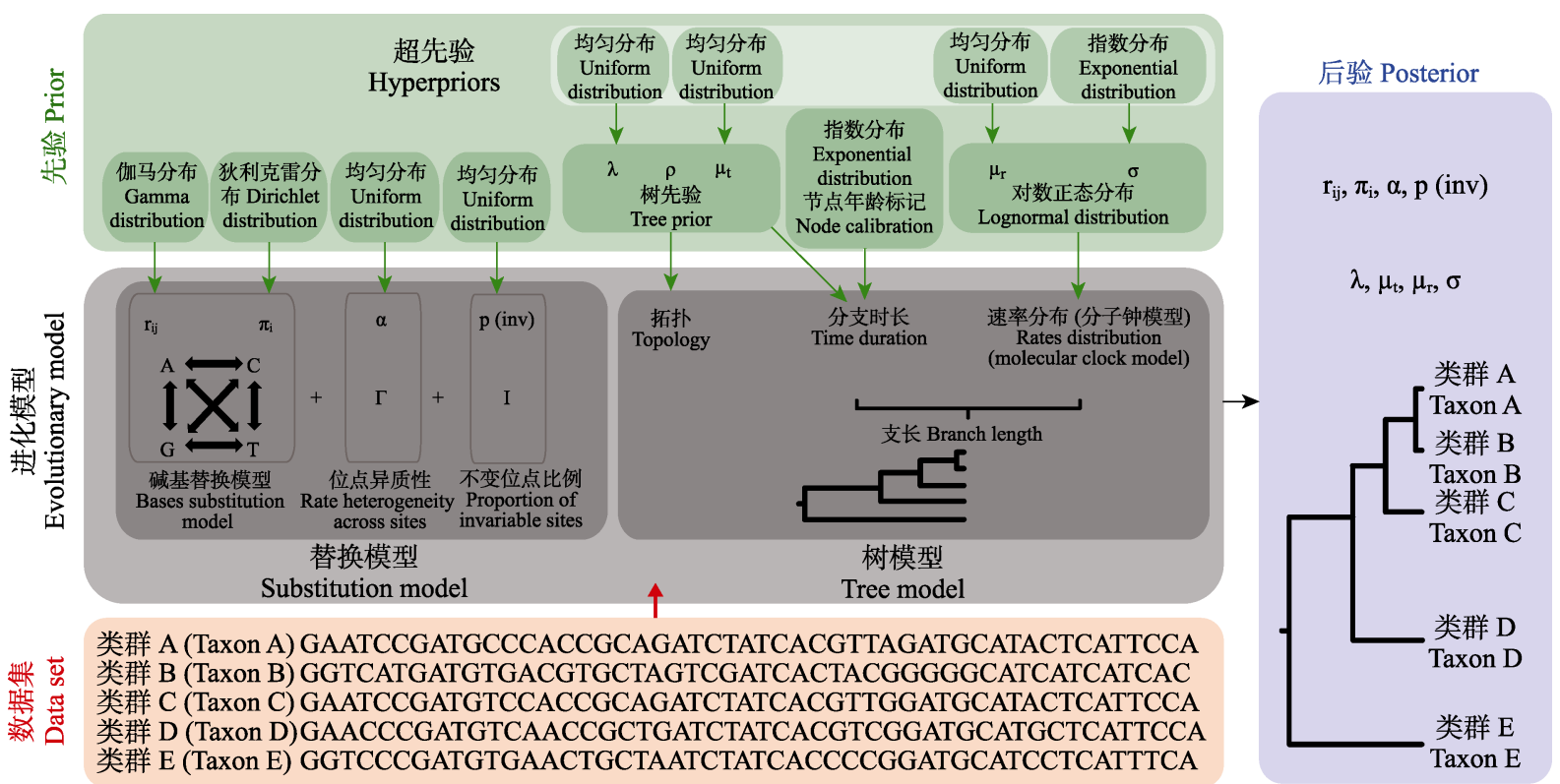

图1 贝叶斯法时间树构建过程示意图(改自: Bromham et al, 2018)。进化模型的参数设置是贝叶斯法系统发育树构建的关键 步骤, 由替换模型和树模型组成。替换模型包括了碱基替换模型(包括碱基转换速率 $r_{\mathrm{ij}}$ 和碱基频率 $\pi_{i}$ 参数)、速率的位点(sites) 异质型(如 $\Gamma$ 分布)以及不变位点的比例p(inv)。树模型可以解构为结构与支长两个组份。结构由树先验决定, 图中使用的是生 灭过程模型, 包含物种生成率 $(\lambda)$ 、物种灭绝率 $\left(\mu_{\mathrm{t}}\right)$ 和取样频率 $(\rho)$ 三个参数。在“两步法”中, 结构还可以来自于树文件的输 入。支长由分支时长与速率分布共同决定。分支时长一方面受树先验的影响, 另一方面与节点年龄标记密切相关; 速率分 布即分子钟模型, 决定了进化速率在不同支上的分布格局。数据集与进化模型共同计算得到各参数的后验及时间树。

Fig. 1 Schematic diagram of Bayesian time tree construction (modified from: Bromham et al, 2018). The parameter setting of evolutionary models is the key step of constructing phylogenetic tree based on Bayesian method, which is composed of substitution model and tree model. The substitution model includes base substitution model (including base conversion rate $r_{\mathrm{ij}}$ and base frequency $\pi_{i}$ as parameters), site heterogeneity of rate (such as $\Gamma$ distribution) and proportion of invariant sites p(inv). The tree model can be decomposed into two components: structure and branch lengths. The structure is determined by tree priors (in this case the birth and death process model), which includes three parameters: species generation rate $(\lambda)$, species extinction rate $\left(\mu_{\mathrm{t}}\right)$ and sampling frequency $(\rho)$. In the "two-step" method, the structure can also come from the input of the tree file. The branch length is determined by the branch duration and the rate distribution across branches. The branch duration is influenced by tree priors and node age calibration. The rate distribution is recognized as clock models, which determine the distribution pattern of evolution rate on different branches. The data set is applied to the evolutionary model to generate posteriors of each parameter and the time tree.

al, 2015)。尽管在某些情况下，节点标记法并不是最 好的方法(Ronquist et al, 2012), 但由于目前多数类 群的化石记录较为贯乏、形态学特征编码困难等问 题, 节点标记法仍然是目前适用范围最广、最常用的 化石标记方法。此外, 由于目前整合了形态学数据 的系统发育研究得出的关键节点年龄有时过于古 老, 令研究人员难以接受, 其算法的可靠性有待验 证和进一步改进(dos Reis et al, 2015)。因此, 在下文 中我们主要讨论影响节点标记法准确度 (accuracy) 与精确度(precision)的因素以及如何提高物种分化 时间分子定年的可靠性。

\section{2 贝叶斯节点标记方法的主要误差来源}

主流贝叶斯节点标记方法的主要误差来源有 3 个方面: (1)替换速率模型(substitution rates models) 和系统发育树结构(tree topology)引入的误差; (2)分 子钟模型(molecular clock models)引入的误差; (3)节 点年龄标记方式(calibrations)引入的误差。

\section{1 替换速率模型和系统发育树结构引入的误差}

不同类群生物的核酸、蛋白质替换速率不同(类 群世代长度、DNA修复机制不同等), 同一物种的不 同位点的替换速率也不同, 这要求研究者在设置分 子钟模型时需要同时考虑多个维度的位点替换概 率分布。通常来说, 在比较物种之间的基因编码区 核酸序列差异时, 第一位、第二位密码子的突变率 与时间具有良好的共线性，而第三位密码子的突变 率远高于第一、第二密码子, 且与时间之间呈现非 线性关系。Strugnell等(2005)研究发现, 第三密码子 明显存在饱和信号, 表明其结果并不可靠。因此在 研究进化速率变异较大的类群以及类群跨度较大 
的系统发育树时, 可以考虑排除第三密码子。同理, 当一个基因位点或密码子位点的替换速率与其他 位点有显著差异、数值异常, 且对后验概率的整合 产生严重影响时, 可以考虑去除该位点(Ronquist et al，2012), 也可以对各部分数据采取不同的分组 (partitions) 替换速率模型。诸如 PartitionFinder (Lanfear et al, 2012)等软件能够对不同基因、不同密 码子位点的组合进行比较, 每个组合使用不同的替 换速率模型, 从而寻找最合适的分组模型, 其结果 可以直接应用于BEAST、MrBayes等主流贝叶斯分 析软件。

另外, DNA序列中同一位点的4种碱基之间的 替换速率并不相同, 因此出现了许多不同的替换模 型。目前最常用的替换模型是 general timereversible模型家族, 包括JC69模型(Jukes \& Cantor, 1969)、HKY85模型(Hasegawa et al, 1985)、K80模 型(Kimura，1980)、F81 模型(Felsenstein, 1981)和 GTR模型(Tavaré, 1986)等, 其中GTR模型最为复杂, 它允许每一种核酸替换都有不同的替换速率。该家 族其余模型的区别在于不同核酸间的替换速率有 多少是相同的(例如JC模型假设所有替换速率都是 相同的; Jukes \& Cantor, 1969)。位点间异质性(site heterogeneity) 可以体现为伽马分布 (Gamma distribution, $\Gamma$, 假设位点间替换速率符合伽马分 布)、恒定速率(invariable, I, 假设位点间替换速率独 立且恒定) 以及 $\mathrm{I}+\Gamma$ 模型等。不同的模型组合可以被 描述为诸如JC69 $+\Gamma 、 H K Y 85+\Gamma$ 和 $G T R+\Gamma+I$ 等模式。 目前多个软件能够根据数据集对替换模型的适合 度进行检验, 例如JModelTest (Darriba et al, 2012)、

Modelgenerator (Keane et al, 2006)、bModelTest (Bouckaert \& Drummond, 2017)等, 从而得出特定数 据集最优的碱基替换模型或者数据集不同部分碱 基替换模型的最优组合。Shapiro等(2006)使用酵母 和病毒的序列对不同等替换模型、位点异质性模型 和密码子位点模型等组合进行了检验, 结果显示, 考虑了密码子位点异质性的模型往往是最好的, 而 HKY、GTR、I和 $\Gamma$ 模型组合的适合度各有差异, 模 型组合在不同类群中的适合度也不同。

在开展分子钟应用时, 系统发育树相对支长和 结构是最基础的信息, 一般需要利用现存物种的生 物大分子序列信息来构建系统发育树。然而, 在主 流的系统发育树构建方法中, 相较于距离法 (distance methods)、简约法(parsimony methods)和似 然法(likelihood methods), 贝叶斯法能够利用复杂 模型最大程度地考虑系统发育树构建中存在支长 和结构的不确定性, 具有较好的准确度和可靠性 (Beerli, 2006; Yang \& Rannala, 2012)。

利用贝叶斯软件构建时间树时, 树的拓扑可以 来自导入的物种树文件, 也可以来源于集成性贝叶 斯软件(如BEAST; Drummond et al, 2012)的计算结 果, 后者主要包括两类: 一类基于溯祖理论 (coalescent theory), 另一类基于物种形成理论 (speciation theory)。目前常用的树先验设置主要基 于物种形成理论, 包括生灭模型(birth-death model) 和尤尔模型(Yule model)等。在设置树先验时, 由于 不用树先验的假设不同, 可能会引入误差。例如生 灭模型和尤尔模型在苏铁类进化时间框架构建过 程中, 二者得出的分化时间差异明显, 后者得出的 节点年龄普遍更大(Condamine et al, 2015)。

拓扑误差最典型的例子是长支吸引 (long branch attraction, LBA)。长支吸引是系统发育树构 建中常遇到的问题之一(Susko, 2015), 在2000年前 后其陆续被发现存在于真核生物众多类群的系统 发育分析研究中(Reyes et al, 2000; Brinkmann et al, 2005)。如果一棵树的真实情况是有两个外部长支被 一个内部短支连接并隔开, 那么最后结果可能会得 到两个长支聚在一起的错误的树结构。这一现象可 能在利用简约法以及模型过于简单且未考虑位点 替换速率异质性的最大似然法和贝叶斯法建树的 过程中出现(Yang \& Rannala, 2012)。产生这一现象 的原因是, 序列中某些位点产生了多次的替换, 因 此分析的位点越多, 长支吸引现象就越有可能发 生。长支吸引的解决方案之一是, 尽量使用全似然 法(full likelihood method, 包含最大似然法和贝叶 斯法), 使用合理位点替换模型, 并剔除进化速率过 快的位点, 同时比较多次运算产生的系统发育树, 进行交叉检验(Susko, 2015)。因为树的拓扑关乎物 种之间的共祖关系和化石标记点放置等, 所以在进 行节点标记前应确保拓扑的准确性。

\section{2 分子钟模型引入的误差}

不同分子钟模型处理不同分支间进化速率差异 的方式不同，可能影响时间树上的节点年龄。

简单的分子钟模型得出的时间树上，姊妹分支 之间的绝对支长是相等的, 但是它们的相对支长存 
在差异，二者之间的进化速率也存在差异，因此需 要进行相应的优化。依照速率优化方式的不同, 主 流的分子钟模型可以分为严格分子钟(strict clock)、 多速率分子钟 (multi-rate clock) 和松驰分子钟 (relaxed clock; Ho \& Duchêne, 2014)。近年来由于核 酸和氨基酸替换速率在不同位点、不同类群间的差 异问题受到越来越多的关注, 某些特定的松弛分子 钟模型如非自相关对数正态分布松弛分子钟模型 (uncorrelated lognormal relaxed clock, ULRC), 越来 越受到欢迎(Miller \& Bergsten, 2012)。

严格分子钟模型(图2b)规定系统发育树中所有 谱系的替换速率为一个恒定的单一的值, 主要应用 于近缘物种，如同属或序列差异小于 $5 \%$ 的物种 (Yang, 2006)和种下单元之间的系统发育构建, 因 为各分支之间的替换速率变化往往很小 (Brown \& Yang, 2011; Ho \& Duchêne, 2014), 这种情况下严格 分子钟相较松弛分子钟具有更为显著的优势
(Brown \& Yang, 2011)。但对于替换速率变化较大的 类群, 严格分子钟模型的准确度则很低(Wertheim et al, 2010)。多速率分子钟模型允许多于一种、但 少于总物种数的速率设定存在于系统发育树中, 这 意味着部分谱系将共享位点替换速率。在一些多速 率分子钟模型中, 聚在一起的类群具有相同或相似 的替换速率，称为局部分子钟(local multi-rate clock; 图2c); 在另一些多速率模型中, 速率的变化则贯穿 整棵树, 称为离散多速率分子钟(discrete multi-rate clock; 图2d; Ho \& Duchêne, 2014)。

松弛分子钟模型允许每一个谱系具有独特的 替换速率, 考虑了每一个谱系的独立性。该模型要 求给出先验以便对支长进行模拟(branching process prior), 这一过程中常用的有生灭进程模型(同时考 虑物种的生成和灭绝)和尤尔模型(生灭进程模型的 特殊情况，仅考虑物种生成率)等。在很多情况下生 灭进程模型比尤尔模型更符合实际情况, 尤其是在 (a) 时间树 Time tree

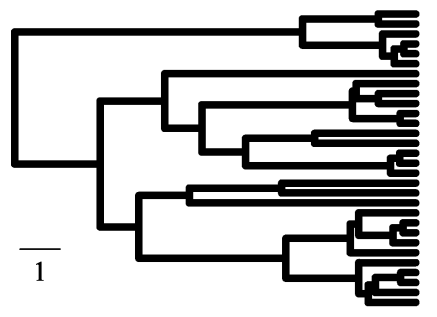

(d) 离散多速率分子钟

Discrete multi-rate clock

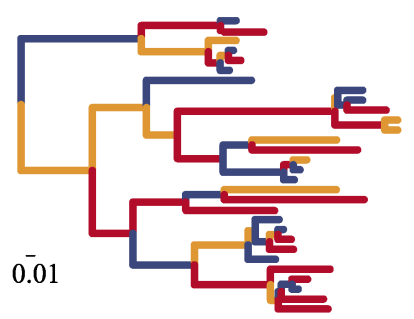

(b) 严格分子钟 Strict clock

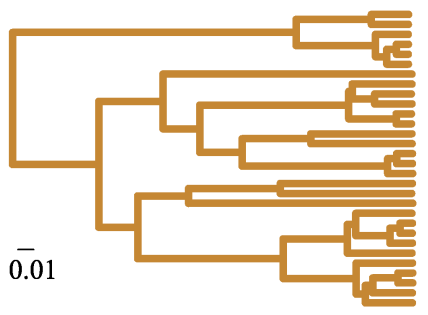

(e) 自相关松驰分子钟 Autocorrelated relaxed clock

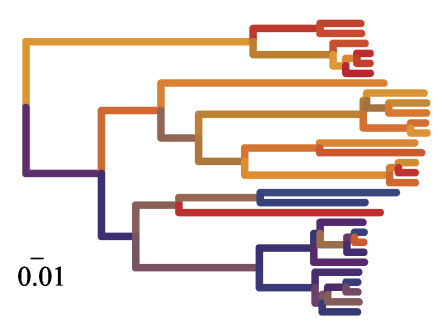

(c) 局部多速率分子钟

Local multi-rate clock

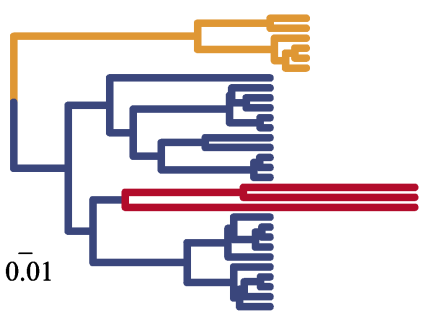

(f) 非自相关松驰分子钟 Uncorrelated relaxed clock

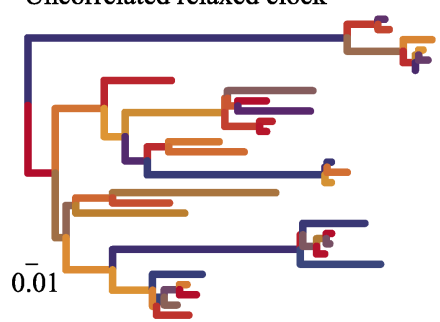

图2 不同的分子钟模型示意图(改自: Ho \& Duchêne, 2014)。图中的6个时间树具有相同的结构, 但由于分子钟模型选择的不 同, 支长有很大差异。(a)未添加分子钟模型的时间树, 比例尺显示了 1 个时间单位。(b)严格分子钟模型, 所有支的速率相等。 (c)局部多速率分子钟, 允许一定量的速率存在, 并根据拓扑的聚类情况设置速率, 同一类群具有相同或相似的速率。(d)离散 多速率分子钟, 允许一定量的速率存在, 不考虑拓扑聚类情况的模型。(e)自相关松弛分子钟, 允许最多等于支数的速率存在, 且邻近支的速率相关。(f)非自相关松弛分子钟, 没有任何对速率的数量、分布格局的限制，是最宽松自由的模型设置。

Fig. 2 Schematic diagram of different molecular clock models (modified from Ho \& Duchêne, 2014). The six time trees in this figure have the same structure, but the branch length varies greatly due to different selection of molecular clock models. (a) The time tree without applying molecular clock model. The scale bar indicates one time unit. (b) In strict molecular clock model, all branches have the same rate. (c) Local multi-rate molecular clock allows a certain amount of rate and sets the rate according to the clustering situation of topology. The related branches have the same or similar rate. (d) Discrete multi-rate molecular clock, which also allows a certain amount of rate, does not consider the topological clustering. (e) The autocorrelated relaxed molecular clock allows the existence of a rate at most equal to the number of branches, and the rate dependence of adjacent branches. (f) The uncorrelated relaxed molecular clock, without any restrictions on the number and distribution pattern of rates, is the most relaxed and free model setting. 
物种灭绝率较高的类群中(Condamine et al, 2015), 并且使用后者得到的节点年龄极有可能比前者大 (Wang \& Mao, 2016)。在贝叶斯框架下, 常见的松弛 分子钟模型又可以分为自相关松弛分子钟 (autocorrelated relaxed clock, 图 2e; Thorne et al, 1998)和非自相关松弛分子钟 (uncorrelated relaxed clock, 又称独立速率分子钟; 图2f; Drummond et al, 2006)两种: 自相关模型中, 相邻谱系之间的替换速 率是相关联的, 体现了谱系之间的生物学特性; 而 非自相关模型中，任何分支之间的替换速率都是独 立的, 能够避免某些快速进化事件对分化年龄估算 带来的影响。自相关松弛分子钟有多种速率分布可 以选择, 常见的有对数正态分布、伽马分布等, 每 个分支上的速率都来自于速率分布, 速率的变化和 时间成正比, 因此自相关松驰分子钟能够体现数量 性状的进化过程。它的缺陷之一在于其对替换速率 的变化范围没有限制, 即替换速率可能随时间不断 提高。为了消除这一不稳定状态, 可以使用内置于 RevBayes (Höhna et al, 2016)等软件中的更加复杂 的 Ornstein-Uhlenbeck 模 型 (Cooper et al，2016; Halliday \& Goswami, 2016)和Cox-Ingersoll-Ross模 型(Lepage et al, 2006; Ho \& Duchêne, 2014)等。非自 相关分子钟每个分支上的速率都来源于速率先验 分布。与自相关分子钟模型不同的是, 其分支之间 的速率相对独立，相互之间没有关联。非自相关分 子钟模型更适用于研究快速进化事件对物种分化 的影响, 并考虑到了系统发育关系与化石标记的不 确定性(Smith et al, 2010)。自相关模型和非自相关 模型各有优缺点, 在条件允许的情况下可分别使用 两种模型构建系统发育树, 并比较其差异, 选择较 优模型。

需要注意的是, 上述各类模型没有优劣之分, 只是各自适用的情况不同(Lee, 2016), 需要通过实 践, 利用客观参数(如贝叶斯因子)进行比较后慎重 选择。除了上述的替代模型和速率先验外, 节点年 龄标记方式也会对系统发育树的构建产生巨大影 响, 节点年龄标记方式设置不合理将直接导致物种 分化时间估算产生误差。

\section{3 节点年齢标记方式引入的误差}

节点年龄标记是节点标记法时间树构建的必 要步骤, 绝对时间信息的来源主要包含: (1)化石标 记 (fossil calibration); (2) 二次标记 (secondary calibration); (3)使用生物地理学事件年龄进行标定 (biogeographic/vicariance calibration)等。

\subsection{1 化石标记}

(1)标记化石选择。分子数据(氨基酸、DNA序 列)只能提供相对的进化时间框架，而绝对分化时 间的最终来源是从相关化石中提取的年龄信息 (Warnock et al, 2017)。分子数据量的增加能够提供 更多信息，从而提高系统发育拓扑和相对分化时间 框架的可靠性, 但物种分化时间估算的准确度和精 密度主要由化石标记的质量决定, 因此化石标记是 物种分化时间估算中最为重要的环节之一(Parham et al, 2012)。

由于化石记录是一个不断挖掘、动态更新的集 合, 因此研究者在使用化石标记时必须格外小心地 篎选和采用可靠的化石记录。随着古生物学的发展, 不断有新的化石被发掘出并被标定年龄, 我们无法 知道某一个化石是否为该类群在地球上存在的最 古老的化石, 而只知道该类群分化产生的时间至少 早于该类群目前已知的、最古老化石的年龄。因此, 使用贝叶斯化石节点标记的主流策略之一, 是使用 目前已知最古老的化石作为一个或多个冠部节点 (crown node)的年龄下限(minimum age constraint), 并往往使用至少一个公认、相对确定的年龄作为根 节点 (root node) 或接近根部的节点的年龄上限 (maximum age constraint), 两者同时作为时间边界, 从而使系统发育树的节点年龄结构逐渐趋于稳定 (Marshall, 2008)。如果没有最大年龄限制, 那么所 得的时间树中, 多数节点的年龄都有高估的潜在可 能性(Claramunt \& Cracraft, 2015)。

虽然实践中通常选择一个类群中已知最古老 的化石, 但在选择分子钟标记化石时, 化石本身的 特性也是需要权衡的重要因素。对于化石的选择主 要考虑两个指标: (i)其年龄是否能够代表一个类群 的最大年龄(代表性, representative); (ii)其分类学特 征是否符合系统发育分析的需求(分类学可分析性, taxonomic solution; Sauquet et al, 2012)。具有代表性 的化石需要能够在该类群刚刚分化出来时就能够 形成、留存到现在并得以被挖掘和发表; 化石的可 分析性则需要化石既拥有足够的、便于聚类的共有 衍征(synapomorphies), 除此之外, 又需要足够的与 其他类群的差异性特征, 才能将化石可靠地放置在 系统发育树中相应的标记节点上。化石的可分析性 
由其完整度、可比对数据集的采样频率等共同决定 (Fikáček et al, 2020)。Fikáček等(2020)依据系统发育 辨析度将化石分为 3 类: (a) 依据形态特征能明显将 其放在进化树上的某一位置, 且无其他备选项; (b) 在进化树上有几个接近的备选位置, 支持度相近并 存在一定冲突(例如由于趋同演化, convergent evolution); (c)在进化树上有众多的备选位置, 后验 概率都很低，表明化石的形态特征信息缺失较多、 其系统发育位置辨析度低。(a)和(b)类化石是节点年 龄标记的较优选择, 在使用系统发育的方法进行辨 析后即可确定位置, 而(c)类化石难以提供有效且准 确的信息。

能够同时满足代表性和可分析性两个特征的 化石是最理想的, 但遗憾的是大多数的化石只能满 足其中一项，这两类化石分别被描述为“古老但有 风险 (old but risky)”和 “年轻但可靠 (young but safe)”。“古老但有风险”的化石的年龄能够代表该类 群已知的最大年龄, 即该类群刚刚分化产生便有化 石并能够较为完整地留存到现在, 然而其分类学特 征往往难以满足系统发育分析的需要(如花粉化石), 这类化石常由于其形态的模糊性(如上文中的 $c$ 类化 石)而只能使用直觉法 (一种较为主观的方法, 见下 文) 确定标记节点, 适合用于标定类群早期分化的 节点(如根节点); “年轻但可靠”的化石往往无法准 确体现(通常会低估)该类群分化产生的时间, 但是 其性状具有很强的可分析性(如上文中的 $\mathrm{a}$ 类化石), 使用上较为安全(并不等于准确), 能够进行更加复 杂的系统发育分析, 例如多数叶、花、果实的化石 (Sauquet et al, 2012)。此外, 对于(b)类化石, 应该将 其放置在不同位置上进行标记, 并将得出的结果进 行比较与讨论。

当上文描述的两个指标不能两全时, 总体来说, 我们倾向于使用“年轻但可靠”的化石进行标记, 因 为其具有很好的系统发育解析度, 不容易产生错置, 尽管其未必能很好地代表整个类群的分化时间。如 果在使用“年轻但可靠”化石的基础上再使用“古老 但有风险”的化石进行标记, 节点年龄会有增大的 趋势(Sauquet et al, 2012)。但这一结果并不代表“古 老但有风险”的化石的放置是错误的, 因为其系统 发育解析的准确性是不可知的。因此, 在进行标记 之前, 应对相关类群的化石进行系统描述, 包括 离群化石(outliers), 在此基础之上, 篮选标定使用
的化石(Parham et al, 2012)。对于“古老但有风险” 的化石, 建议在标记系统发育树基部的节点(如根 节点)时使用, 并设置最大年龄限制(Sauquet et al, 2012)。反之, “年轻但可靠”的化石适合使用在系统 发育树的冠部, 并且其年龄往往小于冠部节点的 实际年龄。

对于一些化石稀缺的类群, 是否选择采用不可 靠的化石进行节点标记是一个值得讨论的问题 (Sanders \& Lee, 2007)。一般情况下, 单个不可靠化 石标记可能会导致一定的误差, 在极端情况下可能 会导致其标记节点的年龄明显大于该节点未标记 的情形。但是, 尽管某些化石的年龄信息并不可靠, 化石标记节点数量的增加仍然能够提高时间树的 可靠性, 尤其是在根节点或近根节点没有化石标记 的情况下(Duchêne et al, 2014; Nie et al, 2020)。因此, 建议同时计算不可靠化石标记和不标记两种情形, 进行比较与讨论, 并阐述采信其一的理由。

(2)标记节点选择。贝叶斯化石节点标记的第一 步是将化石合理地放置于系统发育树中相应的节 点或进化分支上, 主要包括直觉法 (intuitive methods)、衍征法(apomorphy-based methods)和系统 发育法(phylogenetic methods; Sauquet et al, 2012)。 直觉法根据化石类群所能观测到的所有特征, 依靠 直觉在现存类群中寻找与之最为相似的类群。该方 法在早期研究中使用较多, 但由于其判断往往过于 主观, 因此随着学科发展逐渐被弃用。衍征法依照 化石与现存物种之间是否至少有一个共同衍征 (synapomorphy) 以及何时出现共同衍征进行化石位 置放置的推断(Renner, 2005)。具有共同衍征的化石 和现存物种聚为一个单系群, 并以化石年龄作为其 干节点的最小年龄限制(图3)。但由于仅依靠共同衍 征对化石关系进行判断, 该方法也存在一些缺陷, 例如趋同演化(convergent evolution)、衍征数量选择 多少合适以及没有量化的数据分析(例如分子序列 信息和形态学矩阵, morphological matrix) (Sauquet, 2013)。系统发育法则完全依赖系统发育分析重建化 石类群与现存类群之间的关系, 在系统发育分析中 同时使用现存类群和化石类群的数据集, 可以完全 使用形态学数据, 也可以加入现存类群的分子序列 数据进行分析(全证据法)。然而, 这三种化石关系的 评估方法都是建立在化石拥有足够多的差异性形 态特征的基础之上, 否则就无法得出有效的系统发 
育位置信息(Sauquet et al, 2012)。

另一点需要注意的是, 在一棵系统发育树上确 定一个类群的年龄时, 有冠年龄(crown age)与干年 龄(stem age)之分：前者代表该类群现存物种最近 共同祖先的年龄, 而后者代表该类群最近共同祖先 与其姊妹类群的最近共同祖先的年龄。不同的化石 适用于不同的节点年龄类型。如图3所示, 无论是基 于衍征法还是系统发育法进行化石放置, 位于类群 干节点和冠节点之间的化石适合作为类群干节点 的最小年龄限制，位于亚类群干节点和冠节点之间 的化石适合作为类群冠节点的最小年龄限制, 若使 用介于类群干节点和冠节点之间的化石(基于最小 年龄限制)标记冠节点往往导致节点年龄被高估 (Parham et al, 2012)。另外, 因为化石标记点的定位 几乎完全依赖形态学特征(Fikáček et al, 2020), 化 石的完整性是合理确定化石位置的必要条件, 保存 不完整、破碎程度高的化石很可能导致定位不准确, 从而对节点定年产生极大影响(Donoghue et al,

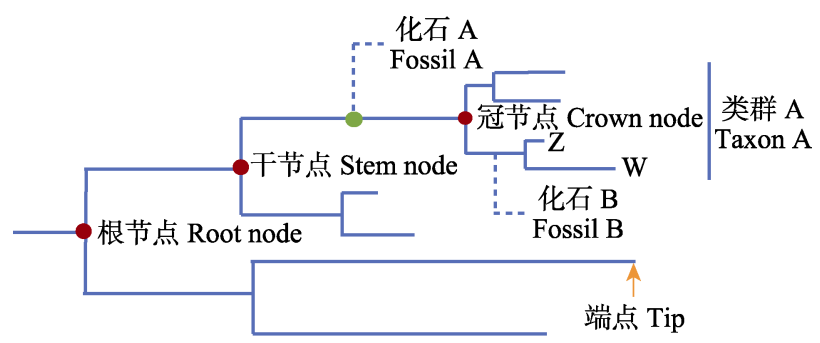

图3 系统发育树中部分术语示意图。图中冠节点的年龄代 表了类群 $\mathrm{A}$ 现存所有物种最近共同祖先的节点, 而干节点代 表了该类群最近共同祖先与其最近缘类群共祖的节点。以衍 征法为例, 图中的化石 $\mathrm{A}$ 与类群 $\mathrm{A}$ 现存物种存在共同衍征, 因此可以作为类群 $\mathbf{A}$ 干节点的最小年龄限制; 而要为类群 $\mathbf{A}$ 的冠节点添加年龄限制, 需要获得与类群 $\mathbf{A}$ 的亚类群具有共 同衍征的化石记录, 如图中的物种 $\mathrm{Z} 、 \mathrm{~W}$ 和化石 $\mathrm{B}$ 所表示的关 系。系统发育法同理。

Fig. 3 Schematic diagram of some terms in phylogenetic tree. The age of the crown node in the figure represents the node of the nearest common ancestor of all species in group A, while the stem node represents the node of the nearest common ancestor of the group A and its nearest related group. Taking the apomorphy-based method as an example, fossil A in the figure has synapomorphies with the extant species of group A, so it can be used as the minimum age constraint for the stem node of group A. To apply age constraint to the crown node of group A, it is necessary to obtain fossil records sharing synapomorphies with subgroups of group A, as indicated by the relationship of species Z, W and fossil B in the figure. The same is true of phylogenetic method.

1989)。在实际操作中, 增加化石标记节点的数量以 及尽量选用接近根节点的化石标记都会改善分化
时间估计的可靠性(Duchêne et al, 2014)。

在研究大尺度系统发育时, 研究人员往往会进 行非随机的、覆盖面广的现存类群取样，但很可能 忽略了一些亚类群(subclade)的取样(如一个大科或 一个大属仅由少数物种代表), 导致得出的取样不 全类群的冠节点年龄实际上为其亚类群的冠节点 年龄(Magallón \& Castillo, 2009), 从而导致年龄低 估的现象。因此现存类群和相关化石的取样不仅应 该使分支取样的覆盖面广, 在选择冠节点进行标记 时还尤其需要对亚系(sublineage)、亚类群进行取样 以保证准确性和代表性。已有研究表明, 化石类群 和现存类群的取样覆盖度偏低都会导致低估节点 年龄(Linder et al, 2005; Sauquet et al, 2012)。

此外, 依据节点之间的拓扑关系将有化石标记 节点的年龄信息附加于无化石节点之上, 也是一种 标记的策略。Barba-Montoya等(2017)使用了三种策 略进行化石标记: (a)对无化石的节点不进行标记; (b)使用有化石节点的年龄上限、下限来标记相邻的 无化石节点; (c)使用有化石节点的年龄上限、下限 来标记所有其他节点, 这样所有的节点都有一对 上下限的设置。结果表明, 方案(c)得到的年龄大于 方案(b), 而(b)大于 (a)。但目前来说, 这类标记策略 的可靠性难以验证, 因此提高化石的采样频率才 是提高定年准确度的根本途径。

\subsection{2 二次标记}

二次标记是在相应类群化石记录贵乏的情况 下, 使用前人系统发育研究中已经得到的节点年龄 或替换速率进行标定的方法。在实际操作中往往选 择一个或多个节点进行二次标记, 或设置前人研究 得出的替换速率作为先验。由于前人研究选择的标 定策略未必一定合理、准确, 再者由于有更多新的 化石被发现、化石年龄得到更新，二次标记得到的 节点年龄往往比化石标记得到的节点年龄年轻, 其 导致的分支替换速率误差甚至能达到 2 到 3 倍之多 (Sauquet et al, 2012)。因此二次标记不是最好的选择, 在相关类群有合适的化石的情况下应优先选择化 石标记。如果确实需要使用二次标记, 应注意选择 从最新发表或者化石标记相对合理的进化时间树 上提取相应节点的年龄。另外, 因为标记节点的增 加有助于提高整体时间计算的准确度(Conroy \& van Tuinen, 2003; Marshall, 2008; Sauquet et al, 2012), 因此可以考虑采用多个节点进行二次标定。 


\subsection{3 使用生物地理学事件年龄进行标定}

在没有合适的化石标记的情况下，使用生物地 理学事件发生的年代进行节点标记也是可行的方 法之一。这往往涉及大陆与板块的运动, 例如澳大 利亚与新西兰的分离可以解释某些物种的隔离分 化事件(Martin \& Dowd, 1993)。如果我们能知道两 个地理区域较为准确的分离时间, 即某些地质事件 年代, 就可以认为分别在两地分布的一对姊妹类群 至少在地质事件发生后产生了分化, 换言之就知道 了二者最近共同祖先节点年龄的下限。然而, 与化 石标记相比, 这种方法无法考虑物种长距离扩散等 由于扩散分布而非隔离分化产生的姊妹类群分化 的情况, 因此这种情况下物种分化的实际时间常晚 于地理事件年龄, 利用地理事件年龄标记得到的类 群的物种分化时间往往比化石标记时间古老(高估) (Sauquet et al, 2012)。

\subsection{4 其他节点标定和替换速率的信息来源}

节点年龄标定的原理在于基于某种证据确定 某一时间节点早于或晚于物种形成事件, 从而对物 种形成时间进行限制。此处所述的证据包括但不限 于化石标记、二次标记和地理隔离标记。其他的设 置方法还有: (1)使用海洋岛屿形成时间作为该地特 有种的年龄上限(Schaefer et al, 2009); (2)使用古 DNA 的年龄作为某一现存后代类群的时间先验 (Korber et al, 2000); (3)使用宿主之间分化的年龄作 为相应严格寄生物种之间分化的年龄上限(Rector et al, 2007)等。此外, 若使用其他研究得出的传代绝
对替换速率作为速率先验, 就可以避免部分时间先 验的设置。例如, 可以通过比较父代和子代之间的 遗传差异计算出世代替换速率，再除以世代时间就 可得出绝对替换速率。但是, 除了模式物种与部分 细菌(Kuo \& Ochman, 2009)、病毒(Vijgen et al, 2005), 绝大多数类群的绝对替换速率往往难以获得 (Sauquet, 2013)。

\subsection{5 节点年龄概率分布的选择}

由于化石记录提供的年龄信息具有不确定性 和时效性(图4), 在利用化石标记设置节点年龄时, 人们倾向于使用带有概率分布 (probability distribution)的先验。概率分布允许节点的时间标记 存在一定的误差, 体现了人类对化石信息的不确定 性的认识。常见的概率分布有均匀分布 (uniform)、 对数正态分布(lognormal)、正态分布(normal)、指数 分布(exponential)和伽马分布(Gamma)等(图5)。在此 基础之上, 可以选择设置严格年龄限制(strict age constraint, 又称硬边界, hard bound)和宽松年龄限 制(soft age constraint, 又称软边界, soft bound; Yang \& Rannala, 2006)。宽松年龄限制允许存在一个较小 的可能性(比如 $5 \%$ 或 $10 \%$ ), 使得节点年龄可以突破 设定的边界(Yang \& Rannala, 2006)。从实际角度出 发, 人们目前获得某一类群最古老化石即为该类群 实际上最古老化石的可能性几乎为零, 宽松年龄限 制以及概率分布曲线可以为研究人员探究更加接 近类群的真实年龄提供途径, 从而避免单一依赖其 年龄下限(dos Reis et al, 2016)。通常对于非根部节
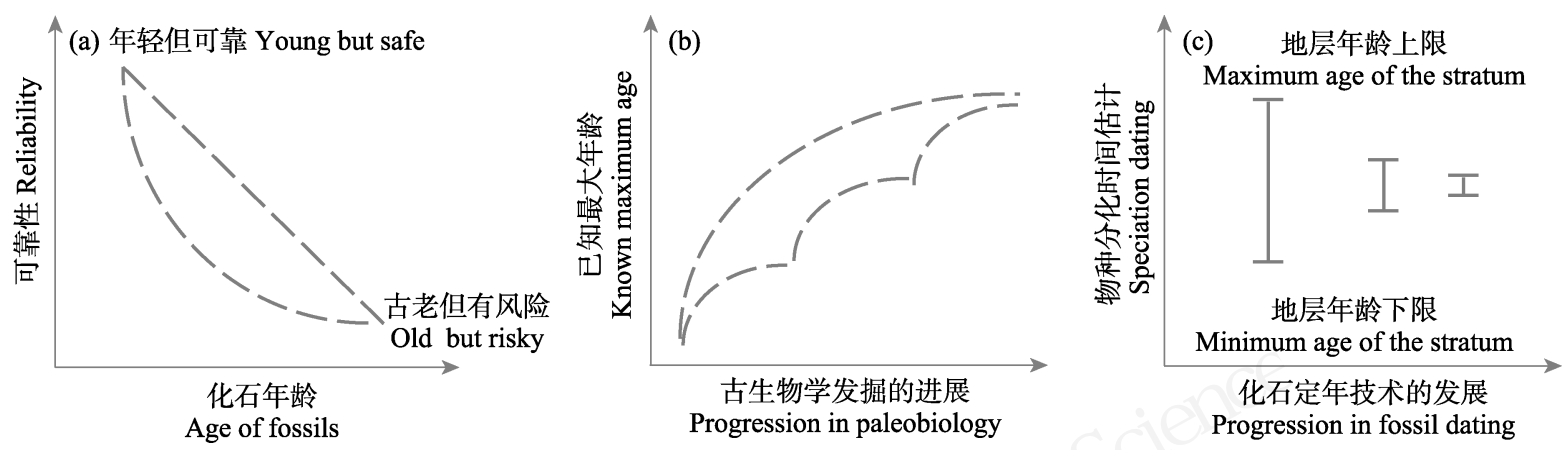

图4 化石年龄不确定性的3种情形。(a)“年轻但可靠”和“古老但有风险”的两种化石在化石年龄和可靠性两个维度上的分布, 其他大多数化石分布于两虚线围成的区域中。(b)某个类群已知最古老化石的年龄随着古生物学发掘过程不断扩展补充、逐 步接近类群真实分化时间的趋势。(c)随着化石定年技术的发展, 化石年龄的精确度不断提升。

Fig. 4 Three cases of fossil age uncertainty. (a) The present of "young but safe" and "old but risky" fossils in two dimensions: age of fossils and reliability. Most of the other fossils are distributed in the area surrounded by the two dotted lines. (b) The age of the oldest known fossil of a certain group tends to be close to the real divergence time of the group as the paleobiological excavation continues to expand. (c) With the development of fossil dating technology, the accuracy of fossil age has been improved. 
点, 化石标记节点年龄的各类先验概率分布都可以 设置一个严格的最小年龄限制(strict minimum age constraint), 表示化石的年龄绝对不小于这一年龄 限制。严格最小年龄限制和宽松最大年龄限制(soft maximum age constraint; Benton \& Donoghue, 2007) 是节点定年中常用的设置组合。近年来, 除了对最 大年龄设置宽松上限以外, 很多研究者还会对最小 年龄也设置宽松下限(Barba-Montoya, 2017; Nie, 2020), 以减少其他因素带来的影响(如化石定年的 误差)。在 MCMCTree 等软件中, 正态分布、伽马 分布、对数正态分布和指数分布的内在逻辑都包含 一个宽松最大年龄限制(图 5c-f), 相应分布模型中 年龄概率分布的参数可以由用户设置。不同的概率 分布对节点年龄的影响有倾向性。

与其他分布相比, 正态分布的特点是其允许双 向的不确定性，即节点年龄可能大于校准信息年龄, 也可能小于校准信息年龄。正态分布尤其适用于某 些利用生物地理学证据进行标记的情况, 因为研究 者往往无法确定遗传分化开始于地理隔离之前还 是之后(Heads, 2005), 正态分布为这一问题的解决 提供了可能。正态分布也适合于二次标记。

相对来讲, 对数正态分布是与古生物学研究现 状较为匹配的一种先验设置(Ho \& Phillips, 2009), 其包含 3 个参数: 均值、标准差和最小年龄限制。如 图 5 所示, 对数正态分布允许节点的年龄概率峰值 大于所用化石的年龄, 认为所用化石即为该类群最 古老化石的可能性几乎为零(将当前化石年龄设置 为最小年龄限制), 这与实际情况是吻合的。但其实 际的均值仍然是未知的, 因此其设置难免主观、武 断。过渡类群的化石适合使用对数正态分布, 因为 这类化石在具有较好的系统发育解析度的同时, 其 特征性状往往被认为是短时间内演化而来的, 因此 能够对该类群的分化时间有较好的预测( 作为均值 进行设置; Slack et al, 2006)。此外, 有研究表明, 基 于分子数据得到的节点年龄是对数正态分布的, 因 此使用对数正态分布导入基于分子数据的二次标 记能够考虑前人数据的误差(Ho \& Phillips, 2009)。

伽马分布的表现形式与对数正态分布十分相 似, 也包含 3 个参数: 形状指数 (alpha)、尺度参数 (beta) 和最小年龄限制。其参数设置和适用条件可以 参考对数正态分布。指数分布有 2 个参数: 均值和最 小年龄限制。它是伽马分布的极端例子, 相比于

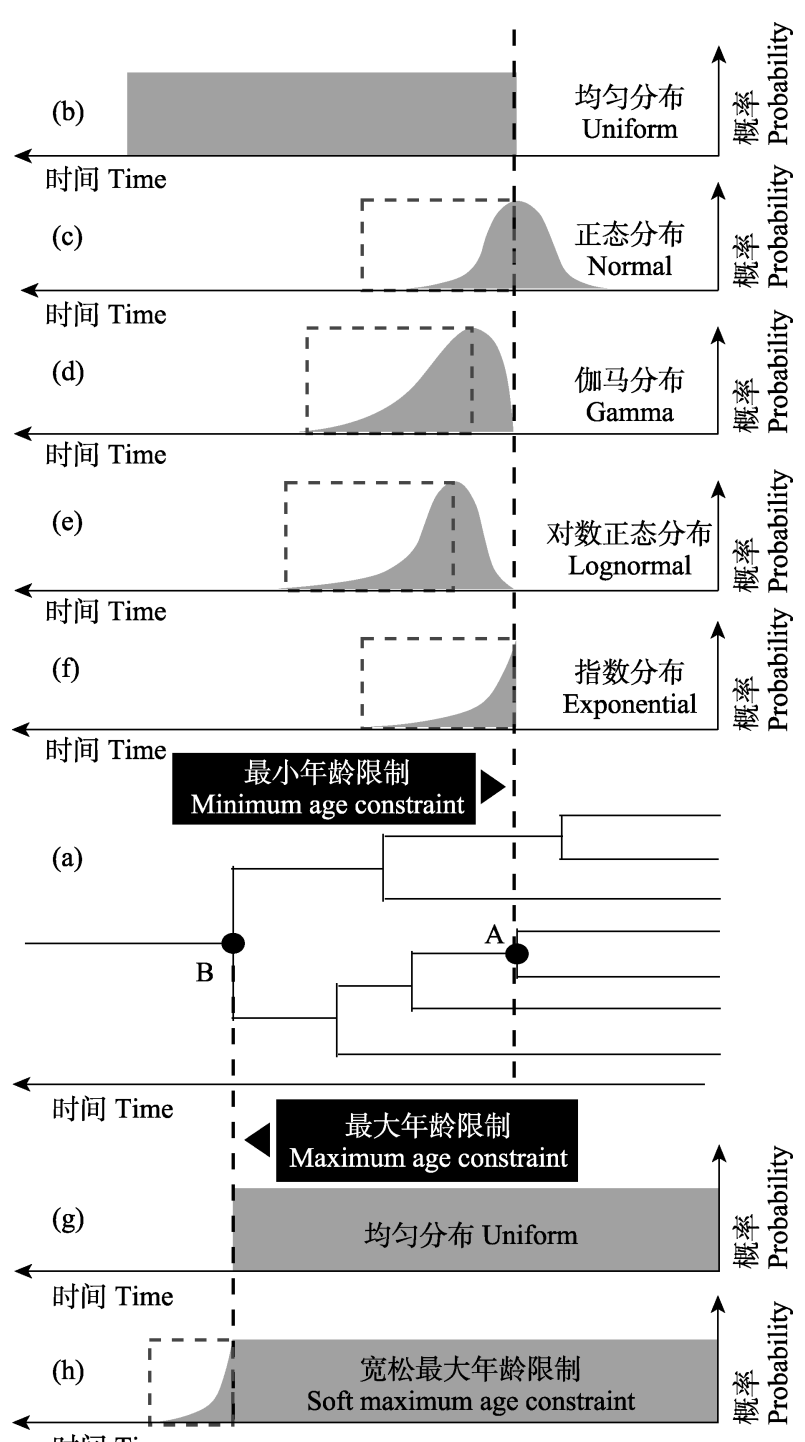

时间 Time

图5 节点年龄的概率分布。在不同节点设置最大或最小年 龄限制, 可以采用不同的概率分布。其中(a)图表示系统发育 树上设置最小和最大年龄限制的两个节点, (b)-(f)强调均 匀、正态、伽马、对数正态和指数分布中的最小年龄限制, 而(g)和(h)则分别表示了均匀分布中的严格和宽松最大年 龄限制(虚线及虚线框)。另外, (c)-(f)中虚线框强调的部分 等同于宽松最大年龄限制。图中的soft maximum/minimum constraint又称软边界(soft bound)。

Fig. 5 Probability distribution of node age. The maximum or minimum age constraint can be set on different nodes, and different probability distribution can be adopted. Where (a) shows two nodes with minimum and maximum age constraint, respectively, in phylogenetic tree; (b)-(f) emphasize the minimum age constraints in uniform, normal, gamma, lognormal and exponential distribution, while ( $g$ ) and (h) represent strict and relaxed maximum age constraints on uniform distribution, respectively. In addition, the part highlighted by the dotted box in (c)-(f) is equivalent to the relaxed maximum age constraint. The soft maximum/minimum constraint in the figure is also called soft bound. 
伽马分布和对数正态分布少了一个参数的设置, 因 此适用于化石信息不太完整的情况。在使用指数分 布时, 研究者相信所使用的化石几乎就是该类群最 古老的化石(Ho \& Phillips, 2009)。

值得注意的是, “年轻但可靠”的化石适合使用 均匀分布，使其实质上仅发挥最小年龄限制的作用; 因为最小年龄现实标记某节点时, 其年龄上限存在 很大的弹性空间，同时施加一个宽松最大年龄限制 (如对数正态分布、正态分布、指数分布和伽马分布 等)可能会导致节点年龄的低估。同时，在根节点可 以考虑使用严格或者宽松最大年龄限制。为了避免 节点年龄无限增大的可能性, 应至少对根节点进行 最大年龄的限制。有研究表明, 在化石标记覆盖面 广的情况下, 根节点的最大年龄限制的小幅波动对 多数节点、尤其是冠部节点的影响十分有限 $(95 \%$ 最 高后验概率密度区间基本重叠; Mao et al, 2012)。最 后, 在实际开展分子钟节点标记时, 强烈建议研究 人员在论文的材料和方法部分提供详细、合理的依 据以说明为什么每个化石标记节点选择相应的节 点年龄概率分布。

\section{3 解读贝叶斯节点标记分子钟估算节点年 龄结果的建议}

利用贝叶斯节点标记分子钟获得时间树之后， 应谨慎解读节点年龄, 建议基于节点年龄的贝叶斯 可信区间(credible intervals)进行相关讨论, 并尽量 避免使用节点年龄的均值进行讨论。一般来说, 分 子钟估算软件产生的结果是由众多先验分布、模型 参数和数据集共同作用得到的, 其中存在着若干未 知参数的影响, 充满了不确定性。因此, 若仅用均 值来讨论节点的年龄并不合适 (Wang \& Mao, 2016)。Crisp 等(2011)的综述阐述了可信区间对于生 物地理学假设检验的重要意义。例如, 假设某一对 姊妹类群的分化是由于某一个地质事件而引发, 则 可比较姊妹类群分化时间的可信区间与地质事件 发生时间二者之间是否存在显著差异。若存在显著 差异, 可以拒绝该假设; 否则, 视为二者存在相关 性，不能拒绝该假设。置信区间(confidence intervals) 和可信区间是频率学派与贝叶斯学派对同一问题 的不同阐释, 其数值上的表现往往是相近的(Chen \& Shao, 1999)。前者不能对参数的概率分布进行评 估, 而后者可以。在模型单一(单峰)时, 贝叶斯可信
区间等同于最高后验概率密度区间 (highest posterior density (HPD) intervals)。

\section{4 分子钟估算软件选择和使用的建议}

使用贝叶斯化石节点标记法进行物种分化时 间估算可以分为两大类: 一类采用先建树后定年的 方法, 即先构建具有拓扑的系统发育树, 然后利用 化石对节点定年得出绝对分化时间树, MultiDivTime、PAML-MCMCTree等软件使用的就 是此类方法, 其优点是速度快 (Ho \& Duchêne, 2014); 另一类方法则同时进行建树和定年, 其运行 速度慢, 但在定年时能充分考虑系统发育树拓扑的 不确定性(Sauquet, 2013), BEAST、MrBayes等软件 主要使用的就是此类方法(各类软件的功能及内置 模型可见参考文献: Ho \& Duchêne, 2014)。化石标记 的各类先验和参数设定引入的不确定性可能引起 系统发育树结构差异，因此在时间和计算资源充裕 的情况下，使用同时建树和定年的方法，可以提高 构建系统发育树结构的准确性; 但在分子数据量或 类群数量过多时, 由于其模型过于复杂, 可能使得 同时建树和定年方法的MCMC 链收敛困难。与之相 比, 只要数据集的信息量足够大, 先建树后定年的 方法不会对系统发育树的拓扑产生影响(Ho \& Phillips, 2009), 因此目前利用全基因组水平数据构 建时间树时通常使用此类软件，如 PAML-MCMCTree等(朱天琪, 2019)。

下面以BEAST v1.10.4软件为例介绍同时建树 和定年类软件的使用。用户需首先在BEAUti软件中 对进化模型参数进行设置, 在BEAUti主界面中可 见到Partitions、Taxa、Tips、Traits、Sites、Clocks、 Trees、States、Priors、Operators、MCMC等分界面， 其具体设置可参考本文各章节给出的建议。需要注 意的是, 在Clocks分界面中, 除了对数、指数等常规 分布外还可以选择“模型平均化”(model averaging), 能够对多个松弛模型进行平均化, 这在对分子钟模 型的选择难以决断时十分实用 (Duchêne et al, 2014)。在Trees界面中可以对树模型和树先验进行 设置, 树模型既可以通过BEAST软件自动计算得出 的树作为起始树(starting tree; 随机选取的树或使用 UPGMA算法的树), 也可以导入NEXUS格式的树 文件作为起始树。在 $\mathrm{MCMC}$ 分界面中除了可以设置 $\mathrm{MCMC}$ 链的长度等参数外, 还可以选择评估边缘似 
然值的方法(marginal likelihood estimation), 用于后 续的模型比较。此后, 将BEAUti生成的XML文件作 为输入文件导入BEAST软件并运算。得到的结果可 以用Tracer软件查看, 包括后验及其他各参数的均 值、标准差、95\%HPD等。

关于BEAST软件使用的入门指南和释疑文档 很多, 例如 BEAST 软件官方网站的使用指南 (Suchard et al, 2018)、福建农林大学高芳銮科学网博 客相关博文(http://blog.sciencenet.cn/home.php?mod $=$ space\&uid=460481)、捷克分子进化培训班 (Workshop on Molecular Evolution, Český Krumlov, Czech Republic, http://evomics.org)于2013年发布的 BEAST详细使用指南等。软件全部为图形界面设置, 操作较为简便, 读者可以选择合适的指南和释疑文 档作为参考。值得注意的是, 近年来BEAST2 (Bouckaert et al, 2014)的使用越来越广泛, 它是 BEAST软件的改写版本, 是一款更加注重模块化及 衍生功能的软件。

随着基因组学与测序技术的发展, 用全基因组 数据建立时间树的方法逐渐得到广泛认可, 这要求 计算机具有较强的计算能力、软件算法更加精巧且 具有较快计算速度。MCMCTree是PAML 4 (Yang, 2007)中的软件包, 与BEAST一样支持多种分子钟 模型、核酸替换模型和年龄先验分布, 支持节点标 记和端点标记的设置, 使用时需要用户提供一棵系 统发育树, 属于先建树后定年类方法。该软件最大 的优势在于可以选择快速近似似然法 (fast approximate likelihood)对时间树进行计算评估，该 方法效率高并且占用计算资源小, 在利用数据量庞 大的基因组进行贝叶斯物种分化时间估计时, 可极 大提高运算速度。与BEAST的可视化界面不同, MCMCTree软件目前没有可视化界面, 需输入代码 进行运算。此外, 由于计算中涉及密码子的 3 个位点, 因此不建议使用氨基酸序列进行计算。软件详细操 作可见PAML4官方说明文件(http://abacus.gene.ucl. ac.uk/software/paml.html)。

\section{5 不同模型、先验设置得到的贝叶斯时间树 的比较方法}

贝叶斯因子(Bayes factor)是检测两个模型间有 无显著差异的统计量。在比较贝叶斯节点分子钟定 年方法中两个不同的模型得出的时间树时, 主流的
比较方法都需要计算贝叶斯因子(Sinsheimer et al, 1996; Suchard et al, 2001), 计算公式如下(Jeffreys, 1935):

$$
B_{10}=\frac{p\left(Y \mid M_{1}\right)}{p\left(Y \mid M_{0}\right)}
$$

其中, 贝叶斯因子 $B_{10}$ 是模型 $M_{1}$ (备择假设, alternative hypothesis) 与模型 $M_{0}$ (零假设, null hypothesis的边缘似然值 (marginal likelihood)之比, $p\left(Y \mid M_{1}\right)$ 和 $p\left(Y \mid M_{0}\right)$ 分别是给定模型 $M_{1}$ 和 $M_{0}$ 后, 数据 集 $Y$ 的边缘似然值(具体数学阐述可见参考文献: Good \& Hardin, 2012)。利用贝叶斯因子检验模型的 参数设置, 相当于回答下列问题: 基于给定数据集 计算时间树时, 零假设(相比于备择假设的特定参 数设置的差异)的可信度(credibility)是否更高? 当 被运用于模型比较时, 给出的数据集在哪个模型中 更加有可能出现?

从 20 世纪 90 年代开始, 对贝叶斯法时间树构建 模型进行评估的统计方法层出不穷, 具有深远影响 的包括调和平均估计量(harmonic mean estimator, HME; Newton \& Raftery, 1994)、赤池信息量准则 (Akaike's information criterion, AIC; Raftery et al, 2006)、路径抽样 (path sampling, PS; Lartillot \& Philippe, 2006)、垫脚石抽样(stepping-stone sampling, SS; Xie et al, 2011)、广义垫脚石抽样(generalized stepping-stone sampling; Fan et al, 2011)等。这些方 法和估计量都致力于解决边缘似然值的复杂计算 问题。HME法与AIC法由于表现不佳(Lartillot \& Philippe, 2006; Xie et al, 2011; Baele et al, 2012a), 其使用频率逐渐降低, 而准确度更高的PS、SS等方 法则为BEAST等主流贝叶斯系统发育分析软件所 采纳运用, 从而得到更好的推广 (Baele et al, 2012b)。

除了主流的贝叶斯因子模型比较法以外, 还可 以利用使用频率相对较低的一些基于其他因子进 行的模型评估方法。例如Wagenmakers等(2020)提出 了支持区间(support interval)的概念, 其所衡量的是 “给定数据集对观测者的预设产生了多大的影响”。 在该方法中, 贝叶斯因子是一个主观设定的参数, 表现了研究者对支持区间呈现范围的预期, 该方法 已应用于bayestestR软件(Makowski et al, 2019)。另 外, Duchêne等(2017)提出了标准差预测距离(stand deviation predictive distance, SDPD)来更准确地衡量 
模型间差异的信号。然而, 相比于主流的比较方法, 这些新兴的贝叶斯模型比较方法有待进一步检验。

有时不同的因子(如贝叶斯因子和支持区间)或 者不同的计算方法对模型的支持倾向有所不同，需 要综合考虑。Van den Bergh等(2021)认为, 当不同评 估参数对模型的评估结果差异较大时, 不必拘泥于 选择某一个模型或参数进行评估, 而可以对两者的 后验概率取均值 (根据边缘似然值加权), 获得联合 后验概率。在现实的系统发育分析中, 依照研究的 需求, 模型与实际数据集并不一定要保证 $100 \%$ 符 合, 而应该在简洁高效与数据的绝对真实性之间寻 求平衡, 从而更好地揭示数据的关键特征(Steel, 2005)。

\section{6 总结}

贝叶斯节点标记法中的不确定性因素有很多, 初学者宜遵循每一个步骤和每一个参数设置都有 据可循(如参考文献支持)的原则来尽量控制不确定 性。我们将提高贝叶斯节点标记法可靠性的建议归 纳为以下几点:

(1)对于不同的替换模型、分子钟模型和先验设 置(尤其是化石标记节点年龄的先验设置)的选择要 慎重, 对于争议较大的情况, 最好分别按照不同的 模型设置和参数设置对实际数据进行分析, 并基于 贝叶斯因子等对结果进行统计分析和横向对比, 选 择最优的模型和参数设置进行最终分析(Ho \& Duchêne, 2014)。在此基础上, 研究者应立足于所研 究的案例, 针对上述争议提出相应意见与观点, 从 而为相应类群乃至相应领域的研究提供改进建议, 进而不断提高节点标记的准确度和精密度(Parham et al, 2012)。例如, 随着分子数据量的增加, 应尽量 使用宽松分子钟模型而避免使用严格分子钟模型 (Ho \& Duchêne, 2014)。其他不同的情况下模型与化 石标记的使用导致的年龄估算偏差, 可参见相关参 考文献(Wang \& Mao, 2016; Sauquet et al, 2012; Shapiro et al, 2006; Wu et al, 2013; Warnock et al, 2017)。

(2)在年龄标记信息准确可靠的基础上，可以 尽量采用多重标记(multi-calibration)进行时间校准。 例如, 对多个节点进行化石标记, 整合使用具有时 间异质性(heterochronous)的序列数据(如古DNA或 不同时期的病毒序列)等(Ho \& Duchêne, 2014)。有
研究表明, 总体上年龄标记数量越多, 节点定年越 准确(Conroy \& van Tuinen, 2003), 但须尽量保证这 些标记年龄是相对准确可靠的(Ho \& Duchêne, 2014)。

(3)在开展化石标记时, 应仔细收集和比较与 目标类群相关的化石, 详细阐述重要的化石类群, 在考虑到主要化石类群的优缺点的基础上, 选择合 适的化石类群开展化石标记。最好能够与古生物学 家和分类学家合作，由其依据已有的、广泛接受的 化石标记原则, 系统、严谨地描述和选择化石类群, 并根据形态学矩阵利用系统发育方法重建现存类 群和化石类群之间的系统发育关系, 从而更合理、 更客观地确定化石标定的节点位置(Parham et al, 2012)。对于引起时间估算争议的关键化石可以分别 计算有(激进)、无(保守)两种情况, 并进行比较与深 入讨论。

(4)应注意更新化石信息(尤其是埋藏点的最新 定年年龄), 并不断更新分子钟定年方法(Parham et al，2012)。若新发现的化石与以前发表的分子钟定 年方法得出的时间树不一致, 不应急于否定分子钟 定年方法(Wilf \& Escapa, 2015), 而应该不断整合最 新发现的、年龄更古老的化石类群作为标记信息， 更新相应类群的时间树(Parham et al, 2012; Wang \& Mao, 2016)。虽然化石记录是绝大多数生物类群获 取绝对分化时间的唯一可靠来源, 但是化石记录本 身是一个不断动态更新、具有时效性的集合(图4), 而特定时间发表的、基于当时化石记录利用分子钟 得出的时间树, 也同样具有时效性; 因此, 新发现 的化石记录对过去化石记录的否定, 只代表对信息 时效性的否定与更新, 而不能将现有化石与以前发 表的、分子钟得出的时间树之间的不一致性, 作为 证据否定分子钟定年方法, 否则便落入了论辩的循 环。

(5)由于各种先验和分子数据的复杂相互作用, 在讨论进化分支分化时间时，应尽量使用贝叶斯可 信区间和最高后验密度区间, 避免使用均值。因为 贝叶斯可信区间和最高后验密度区间代表了整个 贝叶斯分子钟估算过程中的各类不确定性, 而均值 则无法体现。

(6)在条件允许的情况下, 应选择不同类型的 贝叶斯方法(节点标记法、全证据法、化石生灭进程 等)估算时间树并比较不同方法得出的重要节点的 
估算年龄。近年来部分软件支持同时使用节点标记 和端点标记, 并且已有研究表明二者并不冲突且可 优势互补, 从而可以提高节点年龄的准确度和可信 度 (O'Reilly et al, 2015; O'Reilly \& Donoghue, 2016)。需要注意的是, 在软件内开展化石标记时, 可以进行两类方法单独或者混合使用的比较, 尝试 不同组合并择优采纳, 但择优时不宜主观, 应有统 计学数据支持(如计算与比较贝叶斯因子)。

总之, 利用分子钟定年方法估算进化时间框 架的要义在于从不确定性中寻找确定性, 不断更 新化石记录与分子钟定年方法, 从而无限接近于 真理, 即真实发生的、地球生物多样性产生的进化 时间框架。

致谢：本文主要框架出自2018年第三届生物系统 学大会上通讯作者题为“关于提升主流分子钟方法 可靠性的建议”的学术报告。感谢中国科学院微生 物研究所蔡否老师和大会组委会的邀请。

\section{ORCID}

陈囫康 (D) https://orcid.org/0000-0003-4996-0881

李家亮 (D) https://orcid.org/0000-0003-1700-131X

毛康珊 (D) https://orcid.org/0000-0002-0071-1844

\section{参考文献}

Baele G, Lemey P, Bedford T, Rambaut A, Suchard MA, Alekseyenko AV (2012a) Improving the accuracy of demographic and molecular clock model comparison while accommodating phylogenetic uncertainty. Molecular Biology and Evolution, 29, 2157-2167.

Baele G, Li WLS, Drummond AJ, Suchard MA, Lemey P (2012b) Accurate model selection of relaxed molecular clocks in Bayesian phylogenetics. Molecular Biology and Evolution, 30, 239-243.

Barba-Montoya J, dos Reis M, Yang ZH (2017) Comparison of different strategies for using fossil calibrations to generate the time prior in Bayesian molecular clock dating. Molecular Phylogenetics and Evolution, 114, 386-400.

Beerli P (2006) Comparison of Bayesian and maximumlikelihood inference of population genetic parameters. Bioinformatics, 22, 341-345.

Benton MJ, Donoghue PCJ (2007) Paleontological evidence to date the tree of life. Molecular Biology and Evolution, 24, 26-53.

Bouckaert R, Heled J, Kühnert D, Vaughan T, Wu CH, Xie D, Suchard MA, Rambaut A, Drummond AJ (2014) BEAST 2: A software platform for Bayesian evolutionary analysis. PLoS Computational Biology, 10, e1003537.

Bouckaert RR, Drummond AJ (2017) bModelTest: Bayesian phylogenetic site model averaging and model comparison. BMC Evolutionary Biology, 17, 42.

Brinkmann H, van der Giezen M, Zhou Y, De Raucourt GP, Philippe H (2005) An empirical assessment of long-branch attraction artefacts in deep eukaryotic phylogenomics. Systematic Biology, 54, 743-757.

Bromham L, Duchêne S, Hua X, Ritchie AM, Duchêne DA, Ho SYW (2018) Bayesian molecular dating: Opening up the black box. Biological Reviews, 93, 1165-1191.

Brooks DR, Bilewitch J, Condy C, Evans DC, Folinsbee KE, Fröbisch J, Halas D, Hill S, McLennan D, Mattern M, Tsuji LA, Ward J, Wahlberg N, Zamparo D, Zanatta D (2007) Quantitative phylogenetic analysis in the 21 st century. Revista Mexicana de Biodiversidad, 78, 225-252.

Brower AVZ (2018) Statistical consistency and phylogenetic inference: A brief review. Cladistics, 34, 562-567.

Brown RP, Yang ZH (2011) Rate variation and estimation of divergence times using strict and relaxed clocks. BMC Evolutionary Biology, 11, 271.

Chen MH, Shao QM (1999) Monte Carlo estimation of Bayesian credible and HPD intervals. Journal of Computational and Graphical Statistics, 8, 69-92.

Claramunt S, Cracraft J (2015) A new time tree reveals Earth history's imprint on the evolution of modern birds. Science Advances, 1, e1501005.

Condamine FL, Nagalingum NS, Marshall CR, Morlon H (2015) Origin and diversification of living cycads: A cautionary tale on the impact of the branching process prior in Bayesian molecular dating. BMC Evolutionary Biology, $15,1-18$.

Conroy CJ, van Tuinen M (2003) Extracting time from phylogenies: Positive interplay between fossil and genetic data. Journal of Mammalogy, 84, 444-455.

Cooper N, Thomas GH, Venditti C, Meade A, Freckleton RP (2016) A cautionary note on the use of Ornstein Uhlenbeck models in macroevolutionary studies. Biological Journal of the Linnean Society, 118, 64-77.

Crisp MD, Trewick SA, Cook LG (2011) Hypothesis testing in biogeography. Trends in Ecology \& Evolution, 26, 66-72.

Darriba D, Taboada GL, Doallo R, Posada D (2012) jModelTest 2: More models, new heuristics and parallel computing. Nature Methods, 9, 772.

Donoghue MJ, Doyle JA, Gauthier J, Kluge AG, Rowe T (1989) The importance of fossils in phylogeny reconstruction. Annual Review of Ecology and Systematics, 20, 431-460.

Donoghue MJ, Moore BR (2003) Toward an integrative historical biogeography. Integrative and Comparative Biology, 43, 261-270.

dos Reis M, Donoghue PCJ, Yang ZH (2016) Bayesian molecular clock dating of species divergences in the genomics era. Nature Reviews Genetics, 17, 71-80.

dos Reis M, Inoue J, Hasegawa M, Asher RJ, Donoghue PCJ, Yang ZH (2012) Phylogenomic datasets provide both precision and accuracy in estimating the timescale of placental mammal phylogeny. Proceedings of the Royal 
Society B: Biological Sciences, 279, 3491-3500.

dos Reis M, Thawornwattana Y, Angelis K, Telford MJ, Donoghue PCJ, Yang ZH (2015) Uncertainty in the timing of origin of animals and the limits of precision in molecular timescales. Current Biology, 25, 2939-2950.

Drummond AJ, Ho SYW, Phillips MJ, Rambaut A (2006) Relaxed phylogenetics and dating with confidence. PLoS Biology, 4, e88.

Drummond AJ, Suchard MA, Xie D, Rambaut A (2012) Bayesian phylogenetics with BEAUti and the BEAST 1.7. Molecular Biology and Evolution, 29, 1969-1973.

Duchêne DA, Duchêne S, Ho SYW (2017) New statistical criteria detect phylogenetic bias caused by compositional heterogeneity. Molecular Biology and Evolution, 34, 1529-1534.

Duchêne S, Lanfear R, Ho SYW (2014) The impact of calibration and clock-model choice on molecular estimates of divergence times. Molecular Phylogenetics and Evolution, 78, 277-289.

Fan Y, Wu R, Chen MH, Kuo L, Lewis PO (2011) Choosing among partition models in Bayesian phylogenetics. Molecular Biology and Evolution, 28, 523-532.

Felsenstein J (1981) Evolutionary trees from DNA sequences: A maximum likelihood approach. Journal of Molecular Evolution, 17, 368-376.

Fikáček M, Beutel RG, Cai C, Lawrence JF, Newton AF, Solodovnikov A, Ślipiński A, Thayer MK, Yamamoto S (2020) Reliable placement of beetle fossils via phylogenetic analyses-Triassic Leehermania as a case study (Staphylinidae or Myxophaga?). Systematic Entomology, 45, 175-187.

Friston KJ, Lawson R, Frith CD (2013) On hyperpriors and hypopriors: Comment on Pellicano and Burr. Trends in Cognitive Sciences, 17, 1.

Good PI, Hardin JW (2012) Common Errors in Statistics (and How to Avoid Them). John Wiley \& Sons, Hoboken.

Graham TA, Sottoriva A (2017) Measuring cancer evolution from the genome. The Journal of Pathology, 241, 183-191.

Greenwood DR (1991) The taphonomy of plant macrofossils. In: The Processes of Fossilization (ed. Donovan SK), pp. 141-169. Columbia University Press, New York.

Grimm GW, Kapli P, Bomfleur B, McLoughlin S, Renner SS (2015) Using more than the oldest fossils: Dating Osmundaceae with three Bayesian clock approaches. Systematic Biology, 64, 396-405.

Guillerme T, Cooper N (2016) Assessment of available anatomical characters for linking living mammals to fossil taxa in phylogenetic analyses. Biology Letters, 12, 20151003.

Hall BG (2005) Comparison of the accuracies of several phylogenetic methods using protein and DNA sequences. Molecular Biology and Evolution, 22, 792-802.

Halliday TJ, Goswami A (2016) The impact of phylogenetic dating method on interpreting trait evolution: A case study of Cretaceous-Palaeogene eutherian body-size evolution. Biology Letters, 12, 20160051.
Harwood DM, Nikolaev VA, Winter DM (2007) Cretaceous records of diatom evolution, radiation, and expansion. The Paleontological Society Papers, 13, 33-59.

Hasegawa M, Kishino H, Yano T (1985) Dating of the human-ape splitting by a molecular clock of mitochondrial DNA. Journal of Molecular Evolution, 22, 160-174.

Heads M (2005) Dating nodes on molecular phylogenies: A critique of molecular biogeography. Cladistics, 21, 62-78.

Heath TA, Huelsenbeck JP, Stadler T (2014) The fossilized birth-death process for coherent calibration of divergencetime estimates. Proceedings of the National Academy of Sciences, USA, 111, E2957-E2966.

Ho SYW, Duchêne S (2014) Molecular-clock methods for estimating evolutionary rates and timescales. Molecular Ecology, 23, 5947-5965.

Ho SYW, Phillips MJ (2009) Accounting for calibration uncertainty in phylogenetic estimation of evolutionary divergence times. Systematic Biology, 58, 367-380.

Höhna S, Landis MJ, Heath TA, Boussau B, Lartillot N, Moore BR, Huelsenbeck JP, Ronquist F (2016) RevBayes: Bayesian phylogenetic inference using graphical models and an interactive model-specification language. Systematic Biology, 65, 726-736.

Jeffreys H (1935) Some tests of significance, treated by the theory of probability. Mathematical Proceedings of the Cambridge Philosophical Society, 31, 203-222.

Jukes TH, Cantor CR (1969) Evolution of protein molecules. In: Mammalian Protein Metabolism (ed. Munro HN), pp. 21-132. Academic Press, New York.

Keane TM, Creevey CJ, Pentony MM, Naughton TJ, Mclnerney JO (2006) Assessment of methods for amino acid matrix selection and their use on empirical data shows that ad hoc assumptions for choice of matrix are not justified. BMC Evolutionary Biology, 6, 29.

Kimura M (1980) A simple method for estimating evolutionary rates of base substitutions through comparative studies of nucleotide sequences. Journal of Molecular Evolution, 16, 111-120.

Korber B, Muldoon M, Theiler J, Gao F, Gupta R, Lapedes A, Hahn BH, Wolinsky S, Bhattacharya T (2000) Timing the ancestor of the HIV-1 pandemic strains. Science, 288, 1789-1796.

Kumar S (2005) Molecular clocks: Four decades of evolution. Nature Reviews Genetics, 6, 654-662.

Kumar S, Hedges SB (2016) Advances in time estimation methods for molecular data. Molecular Biology and Evolution, 33, 863-869.

Kuo CH, Ochman H (2009) Inferring clocks when lacking rocks: The variable rates of molecular evolution in bacteria. Biology Direct, 4, 35.

Lanfear R, Calcott B, Ho SYW, Guindon S (2012) PartitionFinder: Combined selection of partitioning schemes and substitution models for phylogenetic analyses. Molecular Biology and Evolution, 29, 1695-1701.

Lartillot N, Philippe H (2006) Computing Bayes factors using thermodynamic integration. Systematic Biology, 55, 
195-207.

Laws B (2010) Fifty Plants that Changed the Course of History. Firefly Books, Richmond Hill.

Lee MSY (2016) Multiple morphological clocks and total-evidence tip-dating in mammals. Biology Letters, 12, 20160033.

Lepage T, Lawi S, Tupper P, Bryant D (2006) Continuous and tractable models for the variation of evolutionary rates. Mathematical Biosciences, 199, 216-233.

Li KQ (2015) Quantitative analysis of relationship between absolute evolutionary rates and taxa divergence times. Journal of Biology, 32(2), 70-75, 79. (in Chinese with English abstract) [李可群 (2015) 分子绝对进化速率与物 种分歧时间之间的定量关系. 生物学杂志, 32(2), 70-75, 79.]

Linder HP, Hardy CR, Rutschmann F (2005) Taxon sampling effects in molecular clock dating: An example from the African Restionaceae. Molecular Phylogenetics and Evolution, 35, 569-582.

Liu XF, Zhang AB (2016) The basic principle and application of the molecular clock hypothesis. Acta Palaeontologica Sinica, 55, 393-402. (in Chinese with English abstract) [刘 晓枫, 张爱兵 (2016) 分子钟假说的基本原理及在古生 物等学科中的应用. 古生物学报, 55, 393-402.]

Lu LM, Sun M, Zhang JB, Li HL, Lin L, Yang T, Chen M, Chen ZD (2014) Tree of life and its applications. Biodiversity Science, 22, 3-20. (in Chinese with English abstract) [鲁丽敏, 孙苗, 张景博, 李洪雷, 林立, 杨拓, 陈闽，陈之端 (2014) 生命之树及其应用. 生物多样性, 22, 3-20.]

Magallón S, Castillo A (2009) Angiosperm diversification through time. American Journal of Botany, 96, 349-365.

Makowski D, Ben-Shachar M, Lüdecke D (2019) bayestestR: Describing effects and their uncertainty, existence and significance within the Bayesian framework. Journal of Open Source Software, 4, 1541.

Mao KS, Milne RI, Zhang LB, Peng YL, Liu JQ, Thomas P, Mill RR, Renner SS (2012) Distribution of living Cupressaceae reflects the breakup of Pangea. Proceedings of the National Academy of Sciences, USA, 109, 7793-7798.

Marshall CR (2008) A simple method for bracketing absolute divergence times on molecular phylogenies using multiple fossil calibration points. The American Naturalist, 171, 726-742.

Martin PG, Dowd JM (1993) Using sequences of $r b c \mathrm{~L}$ to study phylogeny and biogeography of Nothofagus species. Australian Systematic Botany, 6, 441-447.

Miller KB, Bergsten J (2012) Phylogeny and classification of whirligig beetles (Coleoptera: Gyrinidae): Relaxed-clock model outperforms parsimony and time-free Bayesian analyses. Systematic Entomology, 37, 706-746.

Morgan GJ (1998) Emile Zuckerkandl, Linus Pauling, and the molecular evolutionary clock, 1959-1965. Journal of the History of Biology, 31, 155-178.

Newton MA, Raftery AE (1994) Approximate Bayesian inference with the weighted likelihood bootstrap. Journal of the Royal Statistical Society: Series B (Statistical Methodology), 56, 3-26.

Nie Y, Foster CSP, Zhu TQ, Yao R, Duchêne DA, Ho SYW, Zhong BJ (2020) Accounting for uncertainty in the evolutionary timescale of green plants through clock-partitioning and fossil calibration strategies. Systematic Biology, 69, 1-16.

O'Reilly JE, Dos Reis M, Donoghue PCJ (2015) Dating tips for divergence-time estimation. Trends in Genetics, 31, 637-650.

O'Reilly JE, Donoghue PCJ (2016) Tips and nodes are complementary not competing approaches to the calibration of molecular clocks. Biology Letters, 12, 20150975.

Parham JF, Donoghue PCJ, Bell CJ, Calway TD, Head JJ, Holroyd PA, Inoue JG, Irmis RB, Joyce WG, Ksepka DT, Patane JSL, Smith ND, Tarver JE, van Tuinen M, Yang ZH, Angielczyk KD, Greenwood JM, Hipsley CA, Jacobs L, Makovicky PJ, Müller J, Smith KT, Theodor JM, Warnock RCM, Benton MJ (2012) Best practices for justifying fossil calibrations. Systematic Biology, 61, 346-359.

Pauling L, Zuckerkandl E (1962) Molecular paleontology. Acta Chemica Scandinavica, 17, S9-S16.

Raftery AE, Newton MA, Satagopan JM, Krivitsky PN (2006) Estimating the integrated likelihood via posterior simulation using the harmonic mean identity. Bayesian Statistics, 8, $1-45$.

Rector A, Lemey P, Tachezy R, Mostmans S, Ghim SJ, Van Doorslaer K, Roelke M, Bush M, Montali RJ, Joslin J, Burk RD, Jenson AB, Sundberg JP, Shapiro B, van Ranst M (2007) Ancient papillomavirus-host co-speciation in Felidae. Genome Biology, 8, 1-12.

Renner SS (2005) Relaxed molecular clocks for dating historical plant dispersal events. Trends in Plant Science, 10, 550-558.

Reyes A, Pesole G, Saccone C (2000) Long-branch attraction phenomenon and the impact of among-site rate variation on rodent phylogeny. Gene, 259, 177-187.

Ronquist F, Klopfstein S, Vilhelmsen L, Schulmeister S, Murray DL, Rasnitsyn AP (2012) A total-evidence approach to dating with fossils, applied to the early radiation of the Hymenoptera. Systematic Biology, 61, 973-999.

Sanders KL, Lee MSY (2007) Evaluating molecular clock calibrations using Bayesian analyses with soft and hard bounds. Biology Letters, 3, 275-279.

Sauquet H (2013) A practical guide to molecular dating. Comptes Rendus Palevol, 12, 355-367.

Sauquet H, Ho SYW, Gandolfo MA, Jordan GJ, Wilf P, Cantrill DJ, Bayly MJ, Bromham L, Brown GK, Carpenter RJ, Lee DM, Murphy DJ, Sniderman JMK, Udovicic F (2012) Testing the impact of calibration on molecular divergence times using a fossil-rich group: The case of Nothofagus (Fagales). Systematic Biology, 61, 289-313.

Schaefer H, Heibl C, Renner SS (2009) Gourds afloat: A dated phylogeny reveals an Asian origin of the gourd family (Cucurbitaceae) and numerous oversea dispersal events. Proceedings of the Royal Society B: Biological Sciences, 
$276,843-851$.

Shapiro B, Rambaut A, Drummond AJ (2006) Choosing appropriate substitution models for the phylogenetic analysis of protein-coding sequences. Molecular Biology and Evolution, 23, 7-9.

Sinsheimer JS, Lake JA, Little RJA (1996) Bayesian hypothesis testing of four-taxon topologies using molecular sequence data. Biometrics, 52, 193-210.

Slack KE, Jones CM, Ando T, Harrison GL, Fordyce RE, Arnason U, Penny D (2006) Early penguin fossils, plus mitochondrial genomes, calibrate avian evolution. Molecular Biology and Evolution, 23, 1144-1155.

Smith SA, Beaulieu JM, Donoghue MJ (2010) An uncorrelated relaxed-clock analysis suggests an earlier origin for flowering plants. Proceedings of the National Academy of Sciences, USA, 107, 5897-5902.

Steel M (2005) Should phylogenetic models be trying to 'fit an elephant'? Trends in Genetics, 21, 307-309.

Strugnell J, Norman M, Jackson J, Drummond AJ, Cooper A (2005) Molecular phylogeny of coleoid cephalopods (Mollusca: Cephalopoda) using a multigene approach: The effect of data partitioning on resolving phylogenies in a Bayesian framework. Molecular Phylogenetics and Evolution, 37, 426-441.

Suchard MA, Lemey P, Baele G, Ayres DL, Drummond AJ, Rambaut A (2018) Bayesian phylogenetic and phylodynamic data integration using BEAST 1.10. Virus Evolution, 4, vey016.

Suchard MA, Weiss RE, Sinsheimer JS (2001) Bayesian selection of continuous-time Markov chain evolutionary models. Molecular Biology and Evolution, 18, 1001-1013.

Susko E (2015) Bayesian long branch attraction bias and corrections. Systematic Biology, 64, 243-255.

Tavaré S (1986) Some probabilistic and statistical problems in the analysis of DNA sequences. Lectures on Mathematics in the Life Sciences, 17, 57-86.

Thomas JA, Welch JJ, Lanfear R, Bromham L (2010) A generation time effect on the rate of molecular evolution in invertebrates. Molecular Biology and Evolution, 27, 1173-1180.

Thorne JL, Kishino H, Painter IS (1998) Estimating the rate of evolution of the rate of molecular evolution. Molecular Biology and Evolution, 15, 1647-1657.

Van den Bergh D, Haaf JM, Ly A, Rouder JN, Wagenmakers EJ (2021) A cautionary note on estimating effect size. Advances in Methods and Practices in Psychological Science, 4, 1-8.

Vijgen L, Keyaerts E, Moës E, Thoelen I, Wollants E, Lemey P, Vandamme AM, Van Ranst M (2005) Complete genomic sequence of human coronavirus OC43: Molecular clock analysis suggests a relatively recent zoonotic coronavirus transmission event. Journal of Virology, 79, 1595-1604.

Wagenmakers EJ, Gronau QF, Dablander F, Etz A (2020) The support interval. Erkenntnis, https://doi.org/10.1007/s10670019-00209-z.
Wang Q, Mao KS (2016) Puzzling rocks and complicated clocks: How to optimize molecular dating approaches in historical phytogeography. New Phytologist, 209, 13531358.

Warnock RCM, Yang ZH, Donoghue PCJ (2017) Testing the molecular clock using mechanistic models of fossil preservation and molecular evolution. Proceedings of the Royal Society B: Biological Sciences, 284, 20170227.

Wasserman L (2004) Bayesian Inference. In: All of Statistics, A Concise Course in Statistical Inference (ed. Wasserman L), pp. 175-190. Springer, New York.

Wertheim JO, Sanderson MJ, Worobey M, Bjork A (2010) Relaxed molecular clocks, the bias-variance trade-off, and the quality of phylogenetic inference. Systematic Biology, 59, 1-8.

Wilf P, Escapa IH (2015) Green Web or megabiased clock? Plant fossils from Gondwanan Patagonia speak on evolutionary radiations. New Phytologist, 207, 283-290.

Wu CH, Suchard MA, Drummond AJ (2013) Bayesian selection of nucleotide substitution models and their site assignments. Molecular Biology and Evolution, 30, 669688.

Xie WG, Lewis PO, Fan Y, Kuo L, Chen MH (2011) Improving marginal likelihood estimation for Bayesian phylogenetic model selection. Systematic Biology, 60, 150-160.

Yang ZH (2006) Computational Molecular Evolution. Oxford University Press, Oxford.

Yang ZH, Rannala B (1997) Bayesian phylogenetic inference using DNA sequences: A Markov Chain Monte Carlo Method. Molecular Biology and Evolution, 14, 717-724.

Yang ZH (2007) PAML 4: Phylogenetic analysis by maximum likelihood. Molecular Biology and Evolution, 24, 15861591.

Yang ZH, Rannala B (2006) Bayesian estimation of species divergence times under a molecular clock using multiple fossil calibrations with soft bounds. Molecular Biology and Evolution, 23, 212-226.

Yang ZH, Rannala B (2012) Molecular phylogenetics: Principles and practice. Nature Reviews Genetics, 13, 303-314.

Zhang C (2019) Molecular clock dating using MrBayes. Vertebrata PalaAsiatica, 57, 241-252.

Zhang Y, Chen ZD (2003) Recent progress of sequences analysis methods in molecular evolutionary biology. Chinese Bulletin of Botany, 20, 462-467. (in Chinese with English abstract) [张原, 陈之端 (2003) 分子进化生物学 中序列分析方法的新进展. 植物学通报, 20, 462-467.]

Zhu TQ (2019) Bayesian molecular dating with genomic data. Scientia Sinica Vitae, 49, 472-483. (in Chinese with English abstract) [朱天琪 (2019) 使用基因组数据进行贝叶斯物 种分化时间估计. 中国科学: 生命科学, 49, 472-483.]

(责任编委：星耀武 责任编辑：时意专) 